\title{
Effect of Group Delay in WCDMA based Pulse Shaping Filter using Discrete-time Eye Scope
}

\author{
A. S Kang \\ Assistant Professor \\ Deptt of Electronics and Comm. Engg \\ SSG Panjab University Regional Centre, \\ Hoshiarpur, Punjab, INDIA
}

\author{
Vishal Sharma \\ Assistant Professor \\ Department of Electronics \& Communication \\ University Institute of Engineering \&Technology, \\ Panjab University Chandigarh
}

\begin{abstract}
The application of signal processing techniques to wireless communications is an emerging area that has recently achieved dramatic improvement in results and holds the potential for even greater results in the future as an increasing number of researchers from the signal processing and communication areas participate in this expanding field. Today, wireless multimedia is moving from vision to reality. Service providers are deploying third generation $(3 \mathrm{G})$ wireless base stations that enable the user to surf the web, check email, conduct videoconferences, and access a wide range of new services from their wireless devices. Underlying 3G innovations, Wideband Code Division Multiple Access (WCDMA) technology is pushing the expectations of wireless networks far beyond the previous limitations of $1 \mathrm{G}$ and $2 \mathrm{G}$. To deliver on the promise of 3G, WCDMA system must handle the greater capacity, higher data rates, and support multimedia standards, while at the same time reduces the size, cost and the power consumption. The large expectations through WCDMA are based on its flexibility for multimedia capabilities and the high capacity. However, the demanded traffic grows rapidly and new capacity enhancements are required in order to satisfy the future needs. The present paper deals with Effect of group delay in WCDMA based pulse shaping filter using discrete-time eye scope.
\end{abstract}

\section{Keywords}

WCDMA, Pulse shaping filter, Group delay

\section{INTRODUCTION}

One of the most challenging issues facing deployment of $3 \mathrm{G}$ technology is how to make the network architectures compatible with each other. New signaling techniques are being designed specially to enhance today's $2 \mathrm{G}$ networks, deliver unprecedented functionality for $3 \mathrm{G}$, and successfully derive the future generation of wireless systems, thus delivering immediate and long term benefits to subscribers. With the architecture of each generation of wireless devices addressed in the development of advance technologies, subscribers can easily evolve their systems without additional network modification, significantly reducing cost and implementing time. The First Generation (1G) and Second Generation $(2 \mathrm{G})$ of mobile telephony were indented for voice transmission. The Third Generation $(3 \mathrm{G})$ is meant for both voice and data applications. The thirst for effective communication and higher bandwidth has led to the evolution of the next generation wireless systems, and newer technologies are being deployed to provide the user with information and entertainment anywhere and anytime. The third generation mobile radio systems (IMT-2000 globally and UMTS in Europe) are becoming a reality today. The network infrastructure is currently being deployed in many countries. Manufacturers, network operators and service providers are now focusing on the development of new services and applications as well as suitable business models to make third generation mobile communication an economic success. One important lesson to be learnt from the development of $3 \mathrm{G}$ is that the potential future services and applications, including the expected user behavior should be taken into account from the very beginning to derive the technical requirements. This approach is essential to enable the economic success of future system. [1-5]

\section{PATH TO THIRD GENERATION 3G}

In many ways, second-generation systems have come about because of fundamental weaknesses in $1 \mathrm{G}$. 1G technology has limited system capacity; they have very little protection against frauds, they are subject to easy eavesdropping, and they have little to offer in terms of advanced features. Secondgeneration systems are designed to address all of these issues. Systems like IS-95, GSM, and IS-136 are much more secure; they also offer higher capacity and more calling features. They are, however, still optimized for voice service and they are not well suited to data communications. In the current environment of the Internet, electronic commerce, and multimedia communications, limited support for data communications is a serious drawback. Although subscribers want to talk as much as ever, they now want to communicate in new ways, such as e-mail, instant messaging, the World Wide Web, and so on. Not only do subscribers want these services, they want mobility too. To provide all of these capabilities means that new advanced technology is required-3G technology. [6]. Third Generation systems started with the vision to develop a single global standard which fulfilled all the demands of a user with high speed data and high quality voice services. In the International telecommunication Union (ITU), third generation networks are called IMT-2000. The leading standards of different countries create a new group called the Third-Generation Partnership Project (3GPP) [7-8]. This group became the driving force behind the development of $3 \mathrm{G}$ standards. Emerging requirements for higher data rate services and better spectrum efficiency are the main driver's identities for the third generation mobile radio systems. There are many leading $3 \mathrm{G}$ systems in current scenario such as WCDMA, CDMA 2000 and Multicarrier CDMA, but the present study deals with WCDMA $[9,10]$. Figure (1) shows the number of subscribers for the first and second generation digital systems from 1995 to 2005. 


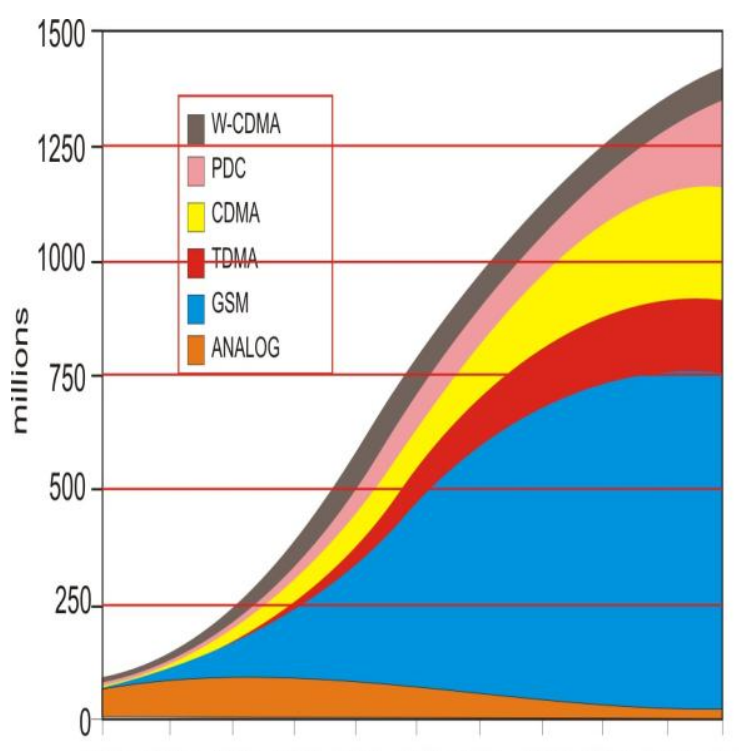

19951996199719981999200020012002200320042005

\section{Figure 1 The Number of Subscribers for the First and Second Generation Systems [9].}

\section{WIDEBAND CDMA}

Wideband Code Division Multiple Access (WCDMA) technology has emerged as the most widely adopted $3 \mathrm{G}$ air interference. Its specification has been created in the $3^{\text {rd }}$ Generation Partnership Project (3GPP), which is the joint standardization project of the standardization bodies from Europe, Japan, Korea, the USA and China [8-10]. WCDMA is considered to be wideband technologies based on the direct sequence spread spectrum transmission scheme, where user information bits are spread over a wide bandwidth by multiplying the user data with quasi-random bits called chips derived from CDMA spreading codes. In order to support very high bit rates (upto $2 \mathrm{Mbps}$ ), the use of a variable spreading factor and multicode connection is supported. The chip rate 3.84 Mcps used to leads a carrier bandwidth of approximately $5 \mathrm{MHz}$. The relatively high bandwidths occupied by CDMA systems are responsible for the significant advantages of CDMA over traditional narrowband systems.

Wideband Code-division multiple access is one of several methods of multiplexing wireless users. In CDMA, users are multiplexed by distinct codes rather than by orthogonal frequency bands, as in frequency-division multiple access. The enhancement in performance is obtained from a Direct Sequence Spread Spectrum (DSSS) signal through the processing gain and the coding gain can be used to enable many DSSS signals to occupy the same channel bandwidth, provided that each signal has its own pseudorandom (signature) sequence [7-9]. Thus enable several users to transmit their information over the same channel bandwidth. This is the main concept of a WCDMA communication system. The signal detection is accomplished at the receiver side by knowing the code sequence or signature of the desired user. Since the bandwidth of the code signal is chosen to be much larger than the bandwidth of the information-bearing signal, the encoding process enlarges or spreads the spectrum of the signal. Therefore, it is also known as spread spectrum modulation. The resulting signal is also called a spreadspectrum signal, and CDMA is often denoted as spreadspectrum multiple access. A tradeoff exists between bandwidth containment in frequency domain and ripple attenuation in time domain. It is this tradeoff of bandwidth containment versus ripple amplitude which must be considered by design engineers, when developing a data transmission system that employs pulse shaping. [9]

\section{TECHNOLOGICAL FEATURES OF PHYSICAL LAYER TRANSMITTER SECTION OF WCDMA SYSTEM}

The following describes the key features of W-CDMA technology to outline the physical layer of the radio interface [10].

- As for the bandwidth, besides the $5 \mathrm{MHz}$ bandwidth, expandability to $10 \mathrm{MHz}$ and

$20 \mathrm{MHz}$ is also secured in order to provide flexibility of operation.

- The chip rate is specified taking into account the hardware feasibility and the Possibility to obtain great spreading gains in the bands used. For instance, when a $5 \mathrm{MHz}$ band is used; the chip rate is set at $3.84 \mathrm{Mcps}$.

- $200 \mathrm{kHz}$ carrier raster enables carrier spacing to be flexible and can very from 4.2 to $5.4 \mathrm{MHz}$. The different carrier spacing can be used to obtain adjacent channel protection depending on the interference scenario and improves spectrum utilization efficiency.

- Figure (2) shows an example for the operator of $15 \mathrm{MHz}$ with three cell layers [10]. 


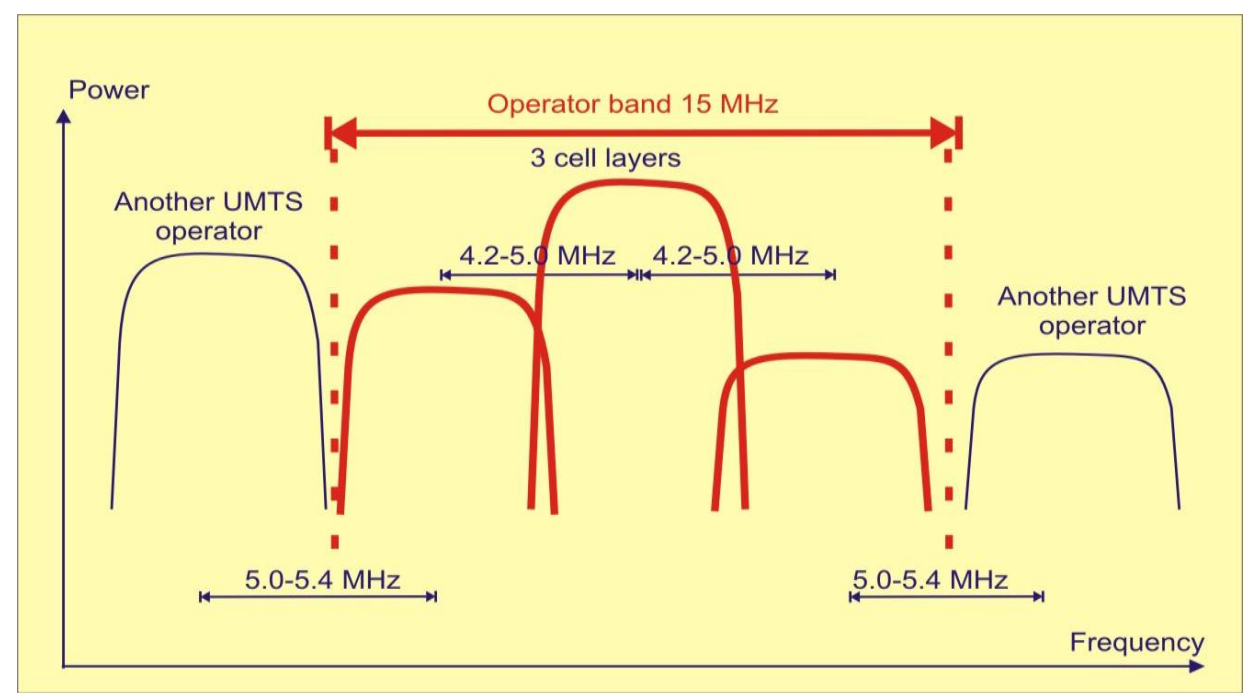

Figure 2 Frequency Utilization with WCDMA [10]

- Convolutional codes are employed for channel coding. Turbo codes that offer better error correction performance are applied for high-speed data transmission [10].

\section{SIMULATION MODEL FOR WCDMA SYSTEM}

The WCDMA communication link proposed in this section is shown in Fig.3. The performance in terms of the Bit Error Rate can be examined for different values of Group Delay D of the pulse shaping filter against a sinusoidal interference. A simulink model based on the matlab 7.3 version will provide the output. The information signal in wideband CDMA system is generated by Bernoulli Binary Generator and the PN sequence is used for spreading the signal at $5 \mathrm{MHz}$ bandwidth. The signal is passed from different parameters block as shown in figure 3 and at the end BER is calculated by comparing the transmitted data and received data. On the basis of above block diagram, a simulation model has been developed by using Matlab Simulink Library as shown in figure 4 .

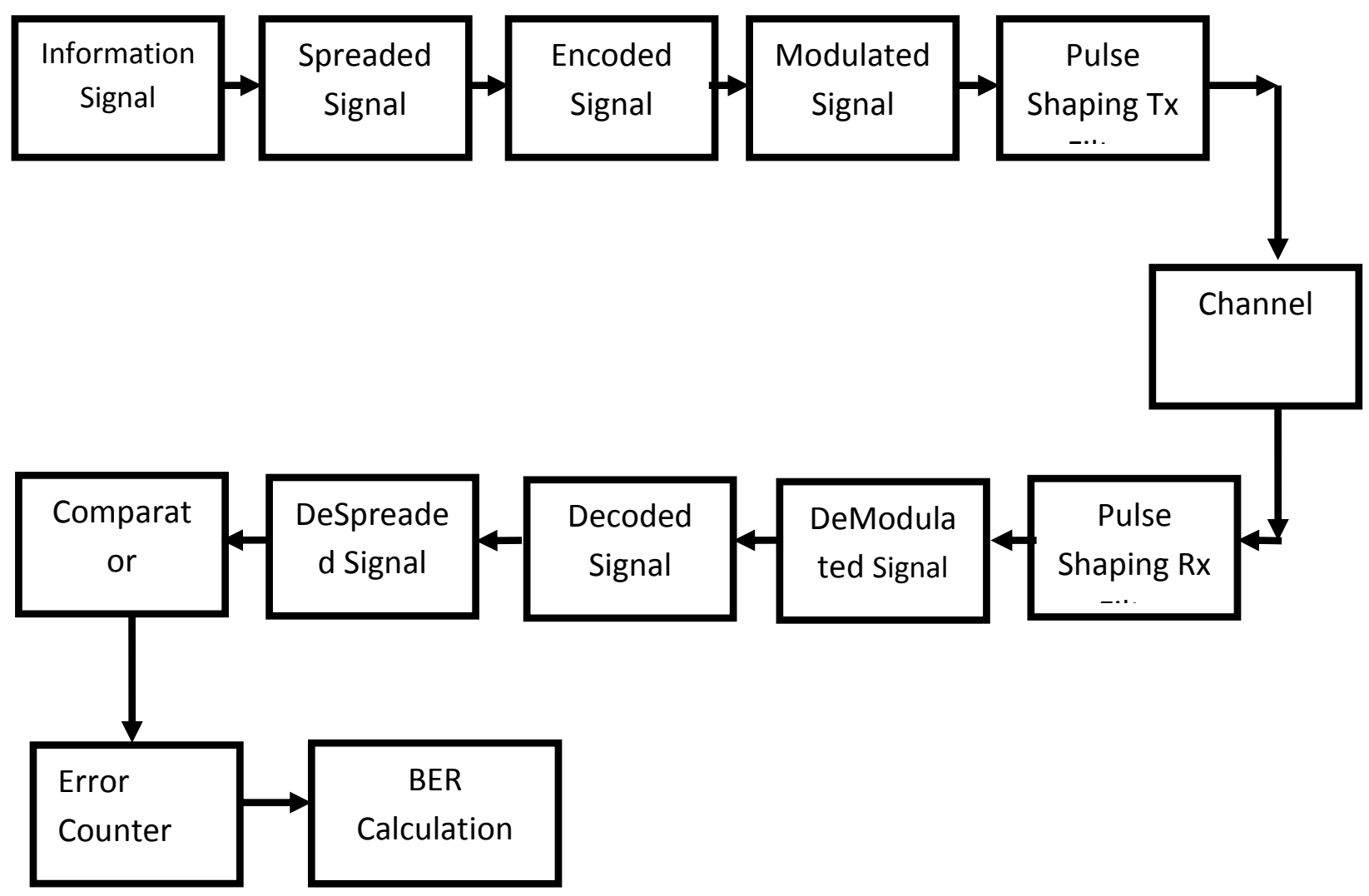

Figure 3 Block diagram for WCDMA System 


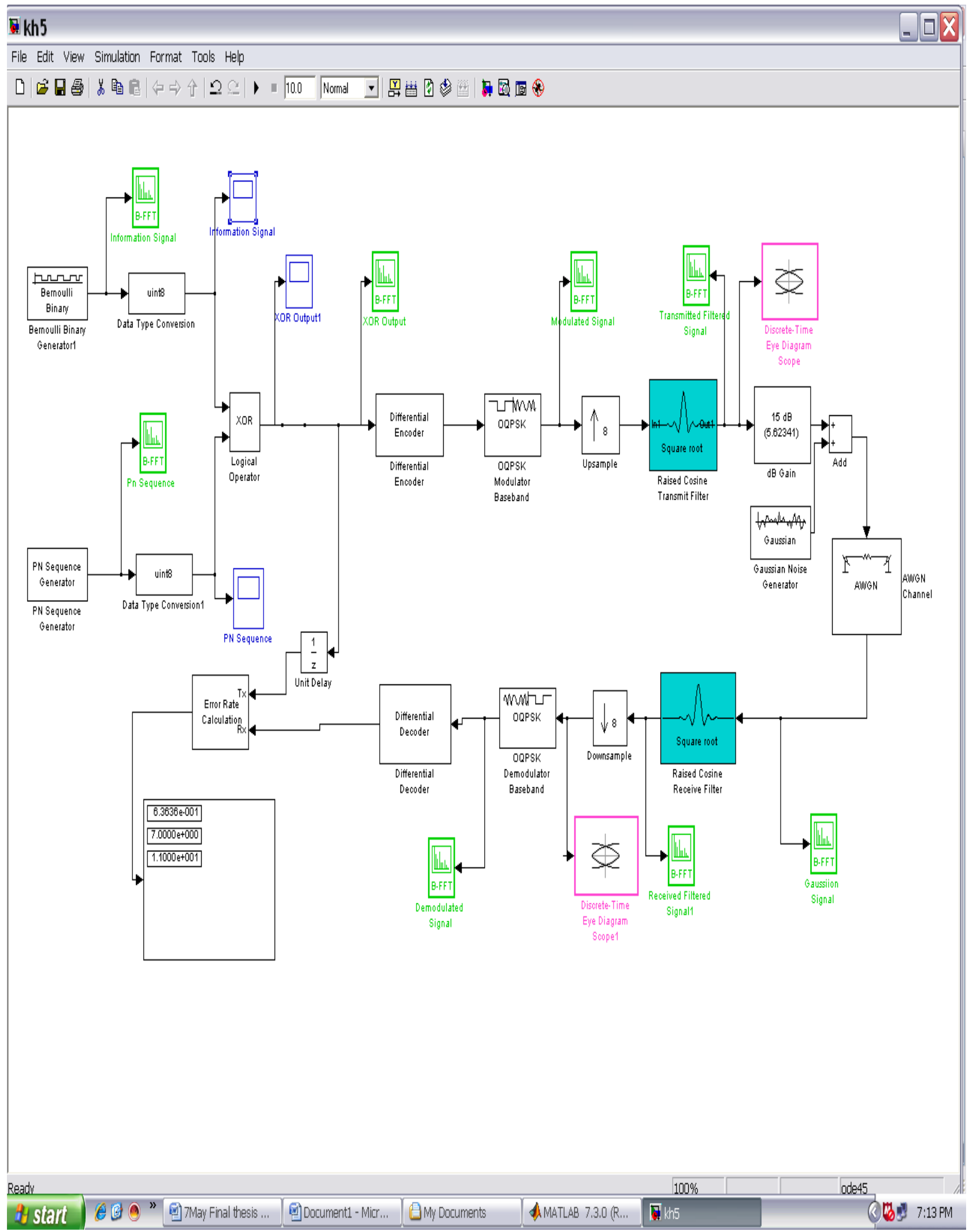

Figure 4. WCDMA based Simulation Model developed for Square Root Raised Cosine Pulse 


\section{ASSUMPTIONS FOR WCDMA SIMULATION MODEL:}

(1)The transmission medium is additive white Gaussian noise.

(2)Effect of Group delay at constant roll off factor alpha and Interpolation factor has been considered

\section{RESULTS AND DISCUSSION:}

Scope as viewed for Information Signal at Input Sample rate $=1 / 64 \mathrm{kbps}$ and Scope as viewed for PN Sequence Generator at Sample Rate $=1 / 3840 \mathrm{Kbps}$ [12-18] The simulation study has also been carried out for different values of D i.e.4,8. The simulation results for BER along with the number of errors and number of bits in each frame

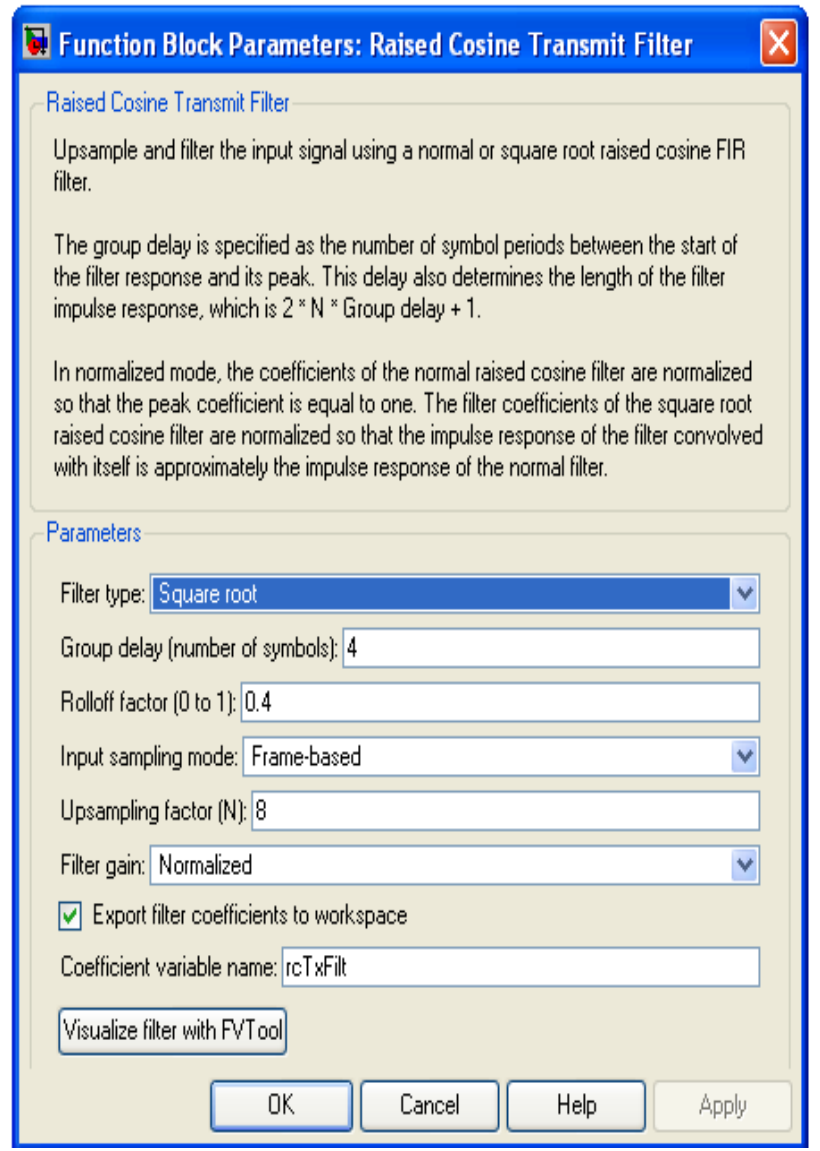

have been obtained. The readings of the simulation model for number of bits, number of errors and Bit Error Rate at

different values of $\mathrm{D}$ have been taken at different time instants during the simulation runs. The parameters of the simulation model are given as below:

$\mathrm{E}_{\mathrm{b}} / \mathrm{N}_{\mathrm{o}}=5 \mathrm{~dB}$

PN Sequence Generator Sample time $=1 / 3840 \mathrm{kbps}$,

Bernoulli Binary Generator Sampletime $=1 / 64 \mathrm{kbps}$ (data services)

Interpolation Factor $\mathrm{M}=5$,

Roll Off Factor $=0.22$ (Optimum) $[8]$

Figure 5 Shows the parameters of pulse shaping filters at group delay $\mathrm{D}=4$

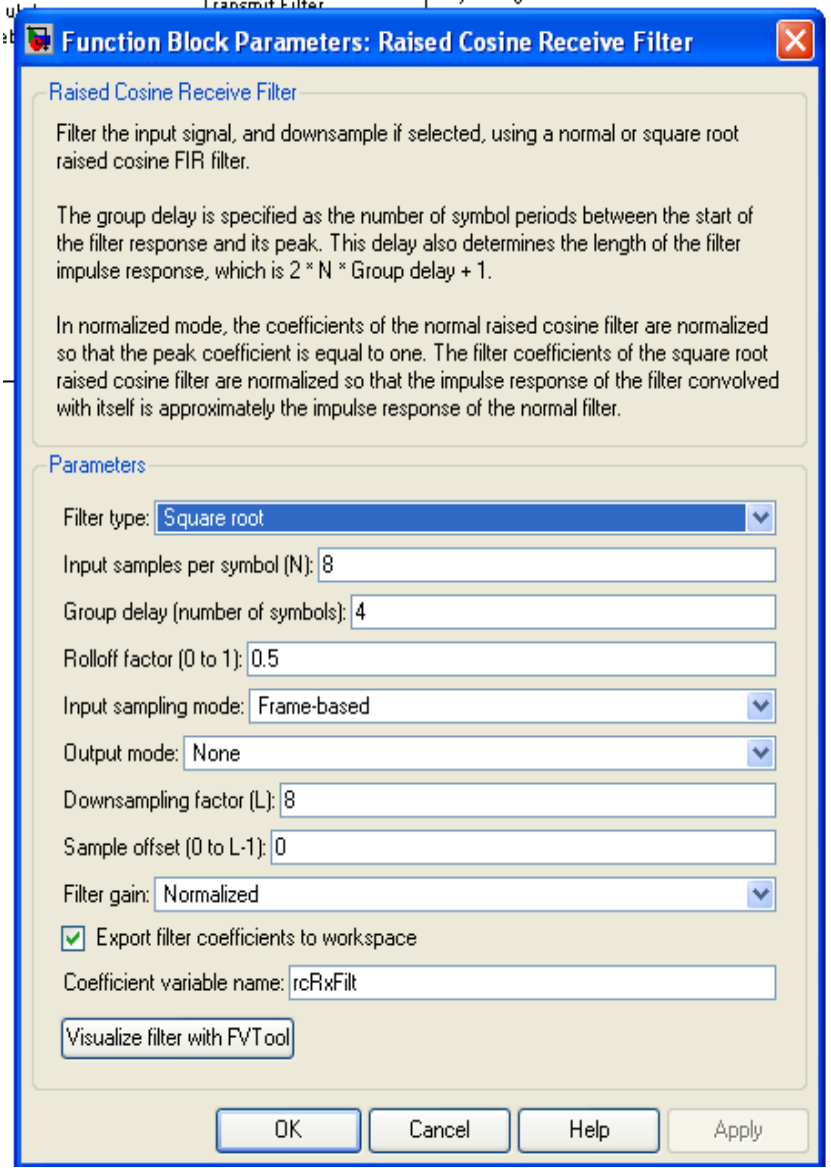

Figure 5 The parameters of pulse shaping filters at group delay $D=4$

Figure 6 shows the time diagram scope , time signal trajectory scope, time signal plot scope for square root raised cosine transmit filter and square root raised cosine receive filter 


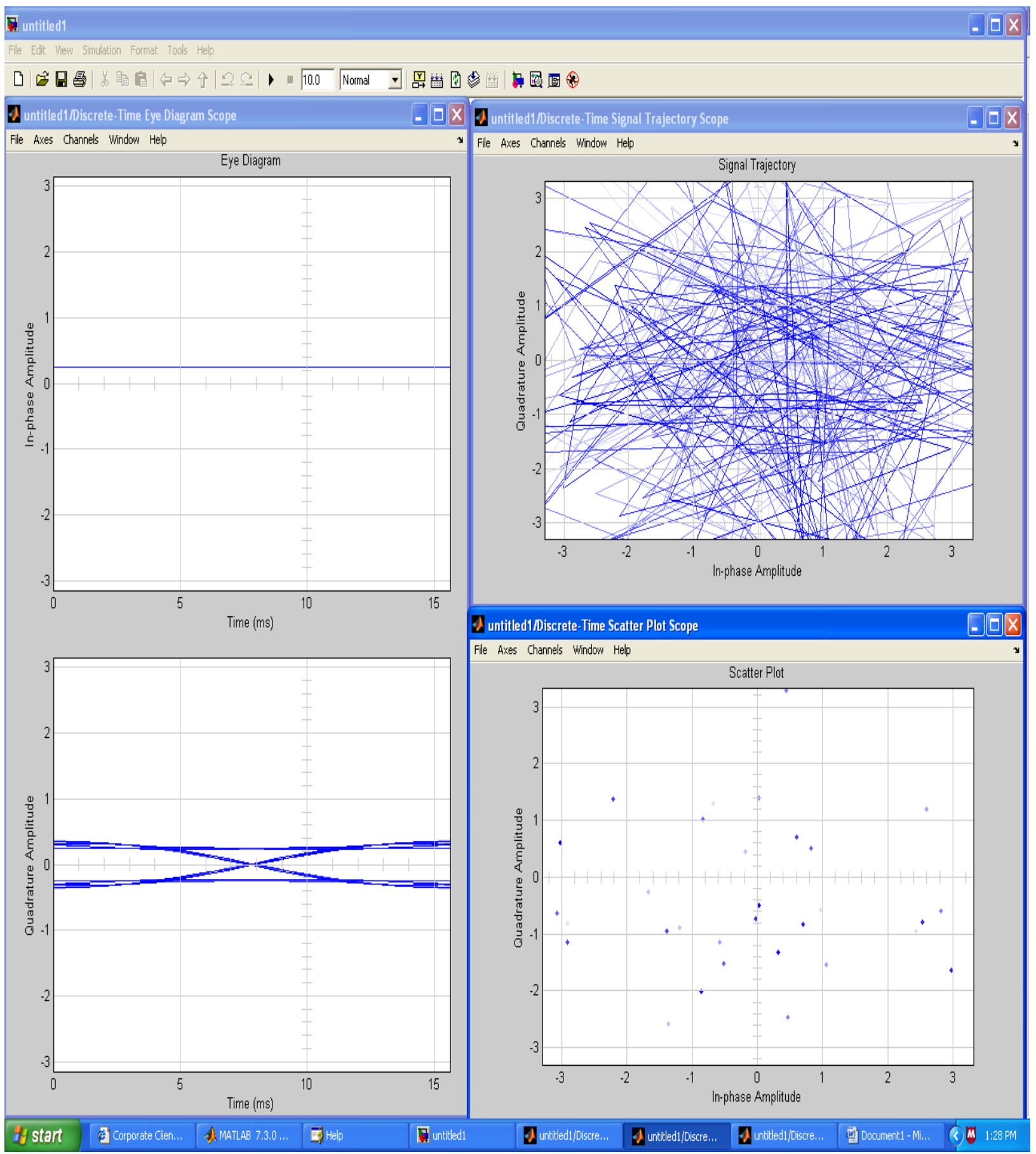

Figure 6 the time diagram scope, time signal trajectory scope, time signal plot scope for square root raised cosine transmit filter and square root raised cosine receive filter

Figure 7 shows the shows the parameters of pulse shaping filters at group delay $\mathrm{D}=8$ 


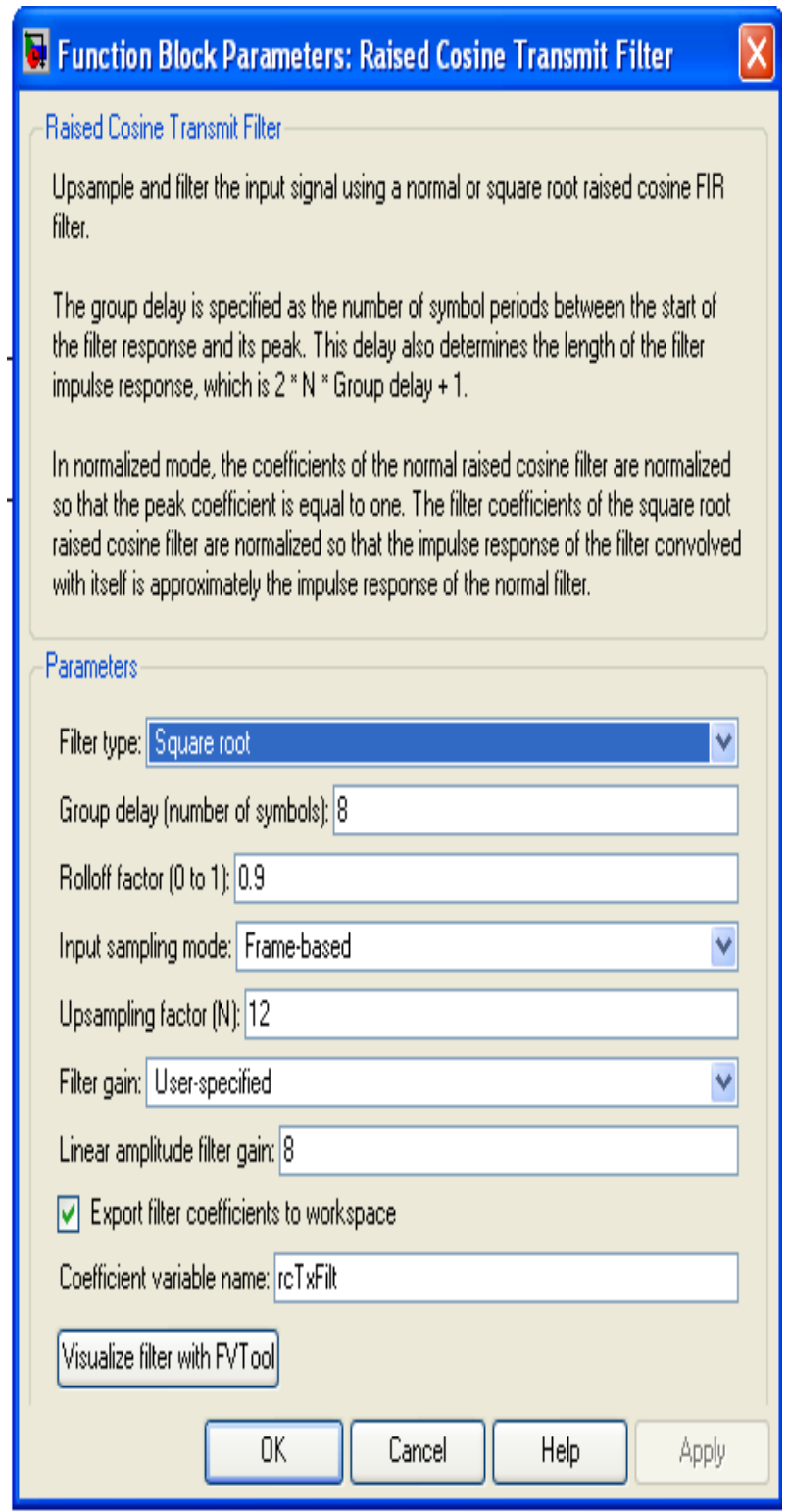

Function Block Parameters: Raised Cosine Receive Filter

Raised Cosine Receive Filter

Filter the input signal, and downsample if selected, using a normal or square root raised cosine FIR filter.

The group delay is specified as the number of symbol periods between the start of the fitter response and its peak. This delay also determines the length of the filter impulse response, which is $2 \times \mathrm{N}$ * Group delay +1 .

In normalized mode, the coefficients of the normal raised cosine filter are normalized so that the peak coefficient is equal to one. The filter coefficients of the square root raised cosine filter are normalized so that the impulse response of the filter convolved with itself is approximately the impulse response of the normal filter.

Parameters

Filter type: Square root

Input samples per symbol ( $N$ ): 8

Group delay (number of symbols): 6

Rolloff factor (0 to 1): 0.5

Input sampling mode: Frame-based

Output mode: Downsampling

Downsampling factor (L): 8

Sample offset (0 to L-1): 0

Filter gain: User-specified

Linear amplitude filter gain: 1

$\square$ Export filter coefficients to workspace

Coefficient variable name: rcRixfilt

Visualize filter with FVTool

OK Cancel Help Apply

Figure 7 the shows the parameters of pulse shaping filters at group delay $D=8$

Figure 8 shows the time diagram scope, time signal trajectory scope, time signal plot scope for square root raised cosine transmit filter and square root raised cosine receive filter at $\mathrm{D}=8$ 


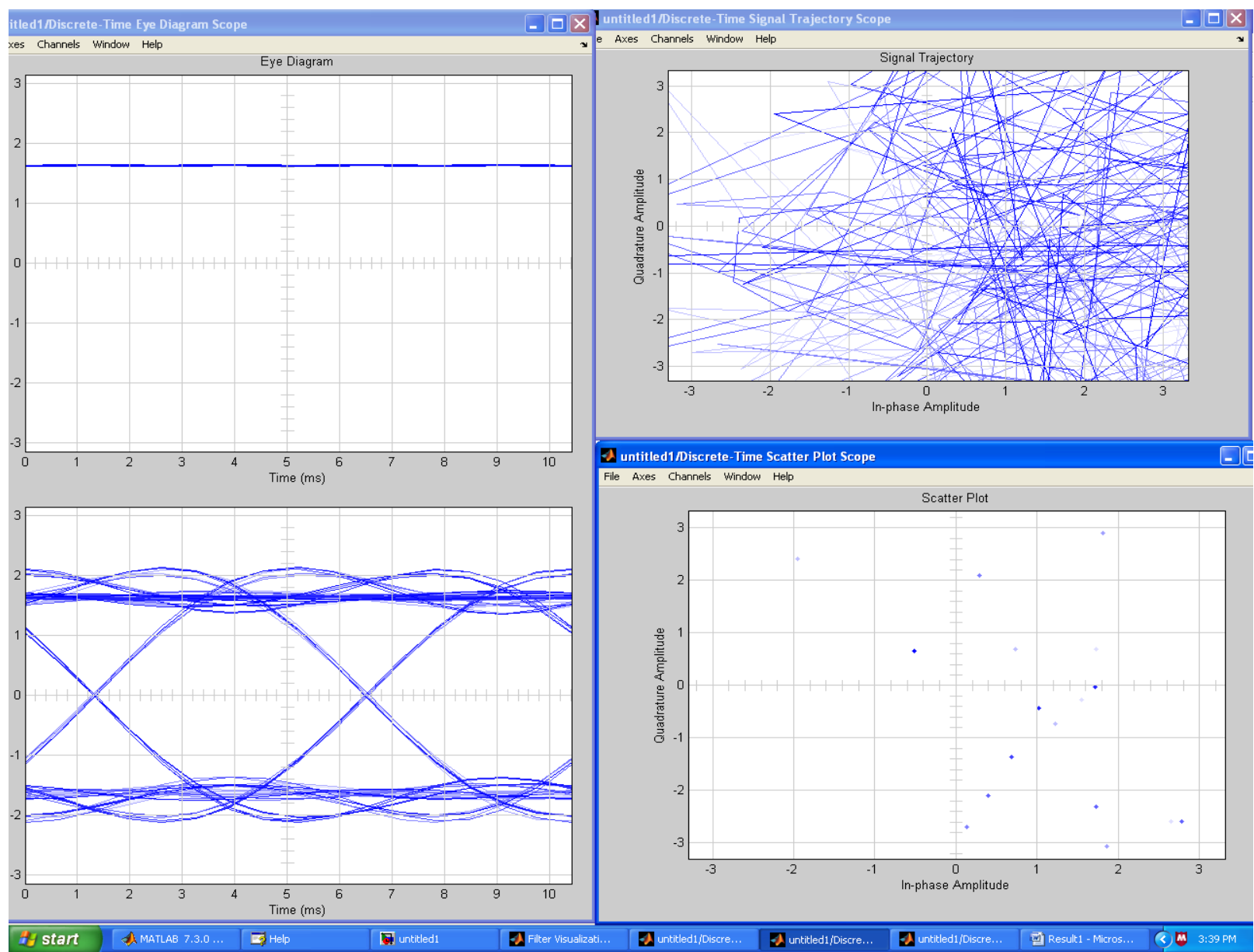

Figure 8 the time diagram scope, time signal trajectory scope, time signal plot scope for square root raised cosine transmit filter and square root raised cosine receive filter at $D=8$

The table 1 provides the comparison of parameters at different values of $\mathrm{D}$.

\begin{tabular}{|l|l|l|}
\hline parameters & Group delay $\mathrm{D}=8$ & Group delay D=8 \\
\hline Opening of eye & small & medium \\
\hline $\begin{array}{l}\text { In phase Signal } \\
\text { trajectory }\end{array}$ & Scattered & More scattered \\
\hline $\begin{array}{l}\text { Quadrature phase } \\
\text { trajectory }\end{array}$ & Scattered & More scattered \\
\hline In phase amplitude & 0.2 & 1.5 \\
\hline
\end{tabular}

Table 1 comparison of of parameters at different values of $\mathrm{D}=4$ and 8.

From the table 1.1 it is clear that complexity of the system increases as the value of group delay is increased from 4 to 8 . Hence an optimum value of $\mathrm{D}$ must be chosen for optimum performance of the communication system.

\section{CONCLUSION}

Square Root Raised Cosine filter has been analyzed for WCDMA at 5Mhz.The effect of variation of roll off factor, group delay and interpolation factor have been studied. [1921]It is necessary for RF design engineers to select the optimum value of $\mathrm{D}$ under the prevailing environment. The present study shows that opening of eye become more complex as the value of $\mathrm{D}$ is changed from 4 to 8 side lobe tail attenuation occurs more quickly as D is increased from 4 to 8 . Hence the group delay must be controlled to decrease the complexity of the filter.

\section{IMPACT OF STUDY}

The study is useful to improve the performance of WCDMA Network.

1. In the planning of WCDMA Network.

2. To achieve the flexibility in use of data rates in different environments.

3. Design of future cellular mobile communication network. 
4. The proposed WCDMA Simulator can be used for optimization of parameters in various environments, with various mobile distributions and different services.

\section{FUTURE SCOPE OF WORK}

The following points have to be concentrated to extend its application to wide range of future directions:

1. Different multipath fading channels can replace AWGN channel in the model to simulate the system under different mobile radio channels.

2. More influencing parameters can be incorporated in the simulation model using adaptive signal processing.

3. The simulation model can be developed for Tan, Beaulieu and Damen pulse shaping families by incorporating more variables.

4. DSP algorithms can be developed for performance enhancement of WCDMA based wireless system using optimized values of parameters of pulse shaping filters.

5. Simulation study can be extended to different data rates such as $144 \mathrm{kbps}, 384 \mathrm{kbps}$ and $2 \mathrm{Mbps}$.

\section{REFERENCES}

[1] R.M Piedra and A. Frish, "Digital Signal Processing comes of age" IEEE spectrum, vol 33,No.5,70,(May 1996).

[2] J Stevens ,"DSPs in Communication "IEEE Spectrum vol.35,No.9,39-46,(Sept.98)

[3] C. Iniaco and D. Embres," "The DSP decision-fixed or floating?” IEEE Spectrum, Vol.3,No.9,PP 72-74,(1996)

[4] P.M. Grant, "Signal Processing hardware and Software" IEEE signal Processing Magazine,vol13,No.1,PP 8692,(Jan 1992).

[5] G. Giannkis, "Highlights of Signal Processing for communications" IEEE Signal Processing Magazine, vol 16,No.2,PP 14-51,(March,1999).

[6] F.Adachi,"Wireless Past and Future-Evolving Mobile Communication Systems,"IEICE Trans Fundamentals,Vol.E84-A,No.1,pp.55-60,(Jan2001)

[7] H. Holma and A. Toskala, "WCDMA for UMTS, "John Wiley \&Sons.Ltd(2002)

[8] 3GPP - www.3GPP.org

[9] Tero Ojanpera and Ramjee Prasad, "Wideband CDMA for Third Generation Mobile Communications," Artech House, Boston, London,(1998).

[10] Keiji TachiKawa,"W-CDMA Mobile Communication Systems"Wiley Publications,pp-26-28,(2002).
[11] A S Kang, Er Vishal Sharma "Digital Processing and analysis of pulse shaping Filter for wireless Communication", presented at $2^{\text {nd }}$ National Conference (Co -Sponsered by IEEE, Chandigarh Sub Section) On Wireless and Optical Communication WOC-2008 at PEC Chandigarh, pp110-113, 18-19 Dec, 2008.

[12] A S Kang, Er Vishal Sharma "Pulse Shaping Filters in Wireless Communication-A Critical Review", Proceedings of National conference on optical and wireless communication(NCOW-2008, Co-Sponsered by Institution of Engineers(IE), India),DAVIET, Jalandhar (Punjab) ,pp 291-297, November 27-28, 2008.

[13] A S Kang, Vishal Sharma "Spectral analysis of filters in cellular wireless communication systems", International Conference on Systemics, Cybernetics, Informatics, ICSCI-2009, Hyderabad, pp-48-55, January7-10, 2009.

[14] AS Kang, Vishal Sharma IEEE -0196,"Study of Spectral Analysis of Filters in Cellular Communication Systems" by A S Kang, Vishal Sharma in IEEE International advance Computing Conference (IACC09)held at Thapar University ,Patiala,(presented),pp 1934-1938, 6-7March 2009.

[15] AS Kang, Vishal Sharma IEEE -0197,"Simulative Investigation of Pulse shaping in WCDMA Wireless Communication" by A S Kang, Vishal Sharma in IEEE International Advance Computing Conference(IACC09) held at Thapar University ,Patiala(presented),pp 1939-1943,6-7 March2009.

[16] AS Kang, Vishal Sharma "Study of pulse shaping fiters in WCDMA under different interferences", IEEESIBCON2009 (IEEE Siberian Conference on Control and Communications) March2009Russia, pp37-41, 28 March2009.

[17] A S Kang and Vishal Sharma, (2010), "Analysis of Parameters of Square Root Raised Cosine Pulse Shaping Digital FIR filter for WCDMA at 5Mhz" International Journal of Electronics Engineering Research Vol2 (2), pp 253-260.

[18] A S Kang and Vishal Sharma, (2010), "Simulation Study of FIR Filter for Complexity Analysis in WCDMA" International Journal of Engineering Science and Technology Vol2 (4), pp 683-692.

[19] A S Kang and Vishal Sharma, (2010), "Simulation of pulse shaping FIR filter for WCDMA" IUP Journal of Information Technology (in Press June 2010 Issue).

[20] A S Kang and Vishal Sharma (2010),"Analysis of Simulation parameters of pulse shaping FIR filter for WCDMA", International Journal of Advancements in Technology http://ijict.org/ ISSN 0976-4860, Vol 1, No 1 (June 2010) @ IJoAT.

[21] A S Kang and Vishal Sharma (2010),"Analysis of Simulation parameters of pulse shaping FIR filter for WCDMA", International Journal of Advancements in Technology http://ijict.org/ ISSN 0976-4860, Vol 1, No 1 (June 2010) @ IJoAT. 